\title{
On Logics of Transitive Verbs With and Without Intersective Adjectives
}

\author{
Selçuk Topal
}

\author{
Bitlis Eren University, \\ Turkey \\ e-mail:s.topal@beu.edu.tr
}

\begin{abstract}
:
The purpose of this paper is to contribute to the natural logic program which invents logics in natural language. This study presents two logics: a logical system called $\mathrm{R}(\forall, \exists)$ containing transitive verbs and a more expressive logical system $\mathrm{R}(\forall, \exists, I A)$ containing both transitive verbs and intersective adjectives. The paper offers three different set-theoretic semantics which are equivalent for the logics.

Keywords: Logic of natural languages; adjectives; transitive relations; transitive verbs; relational syllogistics.
\end{abstract}

\section{Introduction}

Relational syllogistic theories have been taking place in wide applications of different areas such as in natural language theory and generalized quantifiers [5], [1], [7], [9], [8], [22], in algebraic structures [2], [3], [16], [20], in formal logic [4], [11], [12], [15], [14], [17]. The Aristotelian syllogistic did not touch on the validity of sentences containing transitive verbs. De Morgan presented traditional syllogism within relational facts [6]. De Morgan did not mention syllogisms with binary relations with the intention of transitive verbs. Hartmann and Moss extended syllogism with binary relations with the aim of using transitive verbs [17]. Moss presented a logical study using of intersective adjectives in basic syllogistic [12]. Nikolay and Dimiter presented a system of relational syllogistic, based on classical propositional logic and Stone theory [10].

This paper considers the so-called informative verbs. In its atomic propositions " $Q S+v e r b+$ $Q S$ " and " $Q S+$ verb $+Q P_{1}+$ to $+Q P_{2}$ " where $Q \in\{$ some, all $\}$. These verbs designate actions which can be observed and are not depended on their utterances ('to run', 'to take', etc.). However, there are also the so-called performative verbs. They are carried out only by means of uttering them aloud ('to love', 'to hate', etc.). The syllogistic for performative propositions is first introduced in [18]. In this system, there are examined concepts which have no denotations at all verbs such 'love', 'hate', etc. For these concepts, therefore, we can not define an inclusion relation and we need a novel formal system. Some applications of that new syllogistic are proposed in [18], [19].

The current author of this paper presented algebraic semantics (bounded meet semi-lattice) of 
binary and ternary relational logics by using congruence theory [21]. This paper offers some different semantics for $\mathrm{R}(\forall, \exists)$ and $\mathrm{R}(\forall, \exists, I A)$.

\subsection{Some Explanations on Inference Patterns and Languages of the Logics}

In this paper, we study three different equivalent set-theoretic models for inference patterns of sentences in natural language related to intersective adjective phrases in binary relational (transitive verbs) syllogistics. In this sense, there are two logics $\mathrm{R}(\forall, \exists)$ and $\mathrm{R}(\forall, \exists, I A)$ which is a follow-up the work of Moss [20]. Sentences of the language of $\mathrm{R}(\forall, \exists)$ consist of two quantifiers "for all" ( $\forall)$ and "exists" $(\exists)$, and plural nouns and also transitive verbs, but $\mathrm{R}(\forall, \exists, I A)$ 's also include intersective adjectives. Our approach to sentences with or without intersective adjectives falls in model-theoretic semantics. The interpretation of a phrase such as red cars would be the intersection of the interpretation of "red things" and a set of "car individuals".

English sentences such as "all students love some cleaver teachers" are ambiguous. We use these kinds of sentences in meaning of "there is at least one cleaver teacher who all students love". In this regard, the sentences reflect binary relational perspective directly in our logics. On the other hand, we are not interested in sentence forms of Aristotle's syllogistic which consists of Det $+A$ are (are not) $+B$ where Det is $A l l$ or Some or No, and also $A$ and $B$ are plural nouns but Det $+A+$ transitive $v e r b+$ Det $+B$.

Universal quantifiers entail existential quantifiers in our logics because the interpretation of nouns does not allow to be empty set as is in Corcoran's syllogistic system [5]. Some examples of the inference patterns in our languages as follows:

(i) Some students love all teachers

Therefore, some students love some teachers

(i) Some cleaver students see all teachers

(ii) Some instructive teachers see some janitors

Therefore, some students see some instructive teachers

Inferences in Aristotle's syllogistic let sentences to obtain nouns in their conclusions from different the ones in their premises. Although the plural noun educators is not be contained by the premise (i), it does by the conclusion as can be seen in (I3).

(i) Some students see all teachers

(ii) All teachers are educators

Therefore, some students see some educators

Turning to binary syllogistic $\mathrm{R}(\forall, \exists)$ without Aristotle's, one must make inferences with sentences having the same relations, the same nouns and the same orders both in premises and in conclusions as in example (I1). Under the circumstances, the changes must be situated in quantifiers in derivations of the syllogistics but no changes for nouns and relations. On the one hand, the unchangeability of nouns and relations force the structure $R(\forall, \exists)$ to have equivalence classes (see remarks 2.11 and 3.10). Concerning with binary syllogistic $\mathrm{R}(\forall, \exists, \mathrm{IA})$ without Aristotle's, the plural adjectival noun instructive 
teachers is not be contained by the premise (i) but it is contained by the conclusion as can be seen in (I2). This indicates that if there is an intersective adjectival noun in premises, we may have it in conclusion to restrict inferences by intersective adjectival nouns. This situation induces to force using of equivalence classes within the structure $\mathrm{R}(\forall, \exists, \mathrm{I} A)$. In other words, if there is no intersective adjectival noun in premises, we can not make an inference containing intersective adjectival nouns.

Finally, notice that the set of nouns and relations have countable sizes and all models are finite throughout the paper. Languages of the logics in this paper are not closed under boolean operations and do not have recursion.

\section{The Logic of $R(\forall, \exists)$}

Our syntax starts with a collection $P$ of unary atoms (for nouns) and another collection, $R$ of binary atoms (for transitive verbs). A transitive verb takes a subject and a direct object - shall be interpreted as a binary relation on the universe $M$.

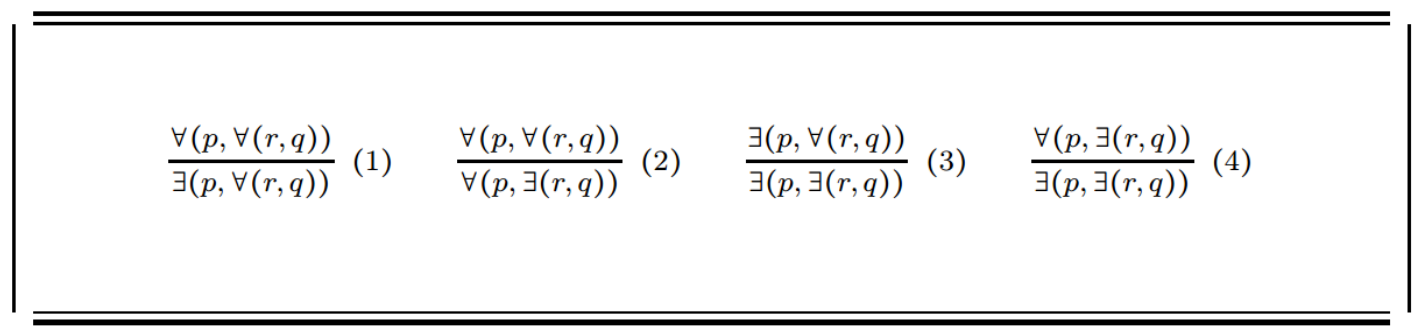

Fig. 1.Rules for $R(\forall, \exists)$

Observation 2.1. An unsound inference:

$$
\frac{\forall(p, \forall(r, q) \quad \exists(p, \forall(r, q))}{\forall(p, \exists(r, q)}
$$

To see the rule is not sound, we construct a counter-model. Suppose that $[[p]]=\left\{p_{1}, p_{2}\right\}$ and $[[q]]=\left\{q_{1}, q_{2}, q_{3}\right\}$ and also $[[r]]=\left\{\left(p_{1}, q_{1}\right),\left(p_{1}, q_{2}\right),\left(p_{1}, q_{3}\right),\left(p_{2}, q_{1}\right),\left(p_{2}, q_{2}\right)\right\}$. Whereas the premises are true in the model, the conclusion $\forall(p, \forall(r, q))$ is false.

\begin{tabular}{|c|c|c}
\hline \hline Syntax & Reading of Syntax & Natural Example \\
\hline$\forall(x, r(\forall, y))$ & All $\mathbf{x} r$ all $\mathbf{y}$ & All students love all teachers \\
\hline$\forall(x, r(\exists, y))$ & All $\mathbf{x} r$ some $\mathbf{y}$ & All students love some teachers \\
\hline$\exists(x, r(\forall, y))$ & Some $\mathbf{x} r$ all $\mathbf{y}$ & Some students see all teachers \\
\hline$\exists(x, r(\exists, y))$ & Some $\mathbf{x} r$ some $\mathbf{y}$ & Some students see some teachers \\
\hline \hline
\end{tabular}

Table 1.Syntax, their natural readings, natural examples

Lemma 2.2. Let $\Gamma$ be a set of sentences in $\mathrm{R}(\forall, \exists)$. The followings hold:

1. $\Gamma \mid-\forall(p, \forall(r, q))$ if and only if (iff) $\forall(p, \forall(r, q)) \in \Gamma$.

2. If $\Gamma \mid+\forall(p, \forall(r, q))$ and $\Gamma \mid-\exists(p, \forall(r, q))$, then $\exists(p, \forall(r, q)) \in \Gamma$. 
3. If $\Gamma \mid+\forall(p, \forall(r, q))$ and $\Gamma \mid-\forall(p, \exists(r, q))$, then $\forall(p, \exists(r, q)) \in \Gamma$.

4. If $\Gamma \mid+\forall(p, \forall(r, q))$ and $\Gamma \mid+\forall(p, \exists(r, q))$ and $\Gamma \mid+\exists(p, \forall(r, q))$ and $\Gamma \mid-\exists(p, \exists(r, q))$, then $\exists(p, \exists(r, q)) \in \Gamma$.

\subsection{Model Construction}

Here, we give some definitions and examples to clarify the paper.

Definition 2.3. $P$ is a set of noun variables, $\forall$ and $\exists$ are quantifiers in language of the logic. $\vec{P}$ is a set which consists of elements which accepted quantifiers in the language as subscript of nouns.

Example 2.4. If $P=\{x, y\}$, then $\vec{P}=\left\{x_{\forall}, y_{\forall}, x_{\exists}, y_{\exists}\right\}$.

Definition 2.5. Let $\Gamma$ be a set of sentences. $P_{\Gamma}$ is the set of nouns occurring in $\Gamma . R_{\Gamma}$ is the set of binary terms in $\Gamma . \vec{P}_{\Gamma}$ is the set of elements of $P_{\Gamma}$ with their quantifiers.

Example 2.6. $\Gamma=\left\{\forall\left(x, \exists\left(r_{0}, y\right)\right), \exists\left(x, \exists\left(r_{1}, y\right)\right), \forall\left(z, \forall\left(r_{1}, h\right)\right)\right\}$.

$$
R_{\Gamma}=\left\{r_{0}, r_{1}\right\}, P_{\Gamma}=\{x, z, y, h\}, \vec{P}_{\Gamma}=\left\{x_{\forall}, z_{\forall}, x_{\exists}, y_{\exists}, h_{\forall}\right\}
$$

Definition 2.7. We define an translation from $\vec{P}$ to $\mathrm{P}(\vec{P})$ as the following:

$$
\begin{aligned}
{[]: \vec{P} \mapsto \mathrm{P}(\vec{P}) } \\
x_{\forall} \mapsto\left\{x_{\forall}, x_{\exists}\right\} \\
x_{\exists} \mapsto\left\{x_{\exists}\right\}
\end{aligned}
$$

Definition 2.8. We define two sets $\left[\vec{P}_{\Gamma}\right]=\left\{[i]\right.$ :for i in $\left.\vec{P}_{\Gamma}\right\}$ and $M^{+} \subseteq \vec{P} \times \vec{P} \times R$

Definition 2.9. Let $\Gamma$ be a set of sentences and $\Gamma_{V e c} \subseteq\left[\vec{P}_{\Gamma}\right] \times\left[\vec{P}_{\Gamma}\right] \times R_{\Gamma}$. We define a translation from $\Gamma$ to $\Gamma_{V e c}$.

$$
\begin{aligned}
& \Upsilon_{V}: \Gamma \mapsto \Gamma_{V e c} \\
& \alpha(p, \beta(r, q)) \mapsto\left(\left[p_{\alpha}\right],\left[q_{\beta}\right], r\right)
\end{aligned}
$$

Please notice that the translation is an one to one correspondence.

Remark 2.10. Note that $\Gamma_{V e c} \subseteq M^{+}$.

Definition 2.11. Two elements $\left(\left[k_{\alpha}\right],\left[l_{\beta}\right], r_{0}\right)$ and $\left(\left[p_{\gamma}\right],\left[q_{\theta}\right], r_{1}\right)$ of $\Gamma_{V e c}$ are in the same equivalence class, if $k=a x$ or $k=x$ and $p=a x$ or $p=x$ and $l=b y$ or $l=y$ and $q=c z$ or $q=z$ and $r_{0}=r_{1}$ where $x, y, z$ are basic nouns.

Remark 2.12. If two elements in $M^{+}$are in the same equivalence class, we will denote two elements 
that first two elements are represented by the same letters and last ones are the same. For instance, $\left(\left[p_{\alpha}\right],\left[q_{\beta}\right], r\right)$ and $\left(\left[p_{\gamma}\right],\left[q_{\theta}\right], r\right)$ are in the same equivalence class because first objects of two elements are denoted by $p$, second ones are $q$ and last ones are $r$.

Definition 2.13. A down-set of element $\left(\left[k_{\alpha}\right],\left[l_{\beta}\right], r_{0}\right)$ of $M^{+} \quad$ is $\quad$ a set $d_{\Downarrow}^{R}\left[\left(\left[k_{\alpha}\right],\left[l_{\beta}\right], r_{0}\right)\right]=\left\{\left(\left[p_{\alpha}\right],\left[m_{\beta}\right], r_{1}\right):\left[p_{\alpha}\right] \subseteq\left[k_{\alpha}\right]\right.$ and $\left[m_{\beta}\right] \subseteq\left[l_{\beta}\right]$ and $\left.r_{0}=r_{1}\right\}$. We also define $d_{\Downarrow}^{R}\left[M^{+}\right]=\left\{d_{\Downarrow}^{R}[i]: i \in M^{+}\right\}$, shortly, $\stackrel{\triangleright}{\Gamma}_{\Gamma}^{+}$.

Definition 2.14. $\left[p_{\alpha}\right] \subseteq\left[k_{\alpha}\right]$ and $\left[m_{\beta}\right] \subseteq\left[l_{\beta}\right]$ and $r_{0}=r_{1}$ iff $\left(\left[p_{\alpha}\right],\left[m_{\beta}\right], r_{1}\right) \subseteq\left(\left[k_{\alpha}\right],\left[l_{\beta}\right], r_{0}\right)$.

Theorem 2.15. $\Gamma \mid-\alpha(p, \beta(r, q))$ iff $\left(\left[p_{\alpha}\right],\left[q_{\beta}\right], r\right) \in \stackrel{\triangleright}{M_{\Gamma}^{+}}$, in other words, $\mathrm{M}_{R}=\left(M_{R},[[]]\right): \Leftrightarrow \mathrm{M}_{R}=(\stackrel{\triangleright}{+}, \in)$.

Proof 2.15. We will prove the theorem on complexity of sentences of $\Gamma$ and elements of $M_{\Gamma}^{+}$. $(\Rightarrow)$ :

(i) Suppose that $\Gamma \mid-\forall(p, \forall(r, q))$. It is clear by Lemma 2.2 .

(ii) Suppose that $\Gamma \mid-\exists(p, \forall(r, q))$ and $\Gamma \mid+\forall(p, \forall(r, q)) . \exists(p, \forall(r, q))$ must be in $\Gamma$ by Lemma 2.2. So, $\left(\left\{p_{\exists}\right\},\left\{q_{\forall}, q_{\exists}\right\}, r\right) \in M_{\Gamma}^{+}$.

(iii) Suppose that $\Gamma \mid-\forall(p, \exists(r, q))$ and $\Gamma \mid+\forall(p, \forall(r, q)) . \forall(p, \exists(r, q))$ must be in $\Gamma$ by Lemma 2.2. So, $\left(\left\{p_{\forall}, p_{\exists}\right\},\left\{q_{\exists}\right\}, r\right) \in \stackrel{\triangleright}{\Gamma}_{\Gamma}^{+}$.

(iv) Suppose that $\Gamma \mid+\forall(p, \forall(r, q))$ and $\Gamma \mid+\forall(p, \exists(r, q))$ and $\Gamma \mid+\exists(p, \forall(r, q))$ and $\Gamma \mid-\exists(p, \exists(r, q))$, then $\exists(p, \exists(r, q)) \in \Gamma$ by Lemma 2.2. Therefore, $\quad\left(\left\{p_{\exists}\right\},\left\{q_{\exists}\right\}, r\right) \in M_{\Gamma}^{+}$. $(\Leftarrow)$ :

(i1) Suppose that $\left(\left\{p_{\forall}, p_{\exists}\right\},\left\{q_{\forall}, q_{\exists}\right\}, r\right) \in \stackrel{\triangleright}{M_{\Gamma}^{+}}$. It is clear by Lemma 2.2.

(i2)Suppose that $\left(\left\{p_{\exists}\right\},\left\{q_{\forall}, q_{\exists}\right\}, r\right) \in M_{\Gamma}^{+}$and $\left(\left[p_{\exists}\right],\left[q_{\forall}\right], r\right) \notin \Gamma_{V e c}$. Then, $\left(\left[p_{\forall}\right],\left[q_{\forall}\right], r\right)$ must be in $\Gamma_{V e c}$ so that $\left(\left\{p_{\exists}\right\},\left\{q_{\forall}, q_{\exists}\right\}, r\right) \in \stackrel{\triangleright}{M}_{\Gamma}^{+}$by the model construction. $\Gamma \mid-\forall(p, \forall(r, q))$ by (i1). Finally, $\forall(p, \forall(r, q)) \mid-\exists(p, \forall(r, q))$ by rule (1) in Figure 1 .

Other proofs are routine.

Theorem 2.16. $\Gamma \mid-\varphi$ iff there exists at least one $\psi$ such that $[\varphi] \subseteq[\psi]$ in $M_{\Gamma}^{+}$.

Proof 2.16. We saw that there is at least one upper set of $\varphi$ to derive it from $\Gamma$ or a sentence $\psi$ due to the definitions $\Upsilon_{V}$ and down-sets in the sufficient condition of Theorem 2.15. 


\section{The Logic of $R(\forall, \exists, I A)$}

Syntax: Our syntax begins with basic nouns $x, y, z \ldots$ by adding intersective adjectives $a, b, c \ldots$ We define the set of nouns, and denote nouns by letters like $n, p$, and $q$, by saying that the basic nouns are nouns, and if $x$ is a noun and $a$ is an intersective adjective, then $a x$ is a noun. We call these nouns of the form $a x$ complex nouns. We do not allow productive predictions which allow to be used more than one adjective in a complex noun such as $a b: x$ where $a$ and $b$ are adjectives and $x$ is a basic noun. One collection $P$ of unary atoms (for nouns) and another collection, $R$ of binary atoms (for transitive verbs). As is in $\mathrm{R}(\forall, \exists)$, verbs will be interpreted as binary relations on the universe $M$.

\begin{tabular}{|c|c|c}
\hline Syntax & Reading of Syntax & Natural Example \\
\hline$\forall(x, r(\forall, y))$ & All $\mathbf{x} r$ all $\mathbf{y}$ & All students love all teachers \\
\hline$\forall(a x, r(\forall, b y))$ & All ax $r$ all by & All cleaver students love all instructive teachers \\
\hline$\forall(x, r(\forall, b y))$ & All $\mathbf{x} r$ all by & All students love all instructive teachers \\
\hline$\forall(a x, r(\forall, y))$ & All ax $r$ all $\mathbf{y}$ & All cleaver students love all teachers \\
\hline$\forall(x, r(\exists, y))$ & All $\mathbf{x} r$ some $\mathbf{y}$ & All students love some teachers \\
\hline$\forall(a x, r(\exists, b y))$ & All ax $r$ some by & All cleaver students love some instructive teachers \\
\hline$\forall(x, r(\exists, b y))$ & All $\mathbf{x} r$ some by & All students love some instructive teachers \\
\hline$\forall(a x, r(\exists, y))$ & All ax $r$ some $\mathbf{y}$ & All students love some teachers \\
\hline$\exists(x, r(\forall, y))$ & Some $\mathbf{x} r$ all $\mathbf{y}$ & All students see all teachers \\
\hline$\exists(a x, r(\forall, b y))$ & Some ax $r$ all by & All cleaver students see all instructive teachers \\
\hline$\exists(a x, r(\forall, y))$ & Some ax $r$ all $\mathbf{y}$ & All cleaver students see all teachers \\
\hline$\exists(x, r(\forall, b y))$ & Some $\mathbf{x} r$ all by & All students see all instructive teachers \\
\hline$\exists(x, r(\exists, y))$ & Some $\mathbf{x} r$ some $\mathbf{y}$ & All students see all teachers \\
\hline$\exists(a x, r(\exists, b y))$ & Some ax $r$ some by & All cleaver students see all instructive teachers \\
\hline$\exists(a x, r(\exists, y))$ & Some ax $r$ some $\mathbf{y}$ & All cleaver students see all teachers \\
\hline$\exists(x, r(\exists, b y))$ & Some $\mathbf{x} r$ some by & All students see all instructive teachers \\
\hline \hline
\end{tabular}

Table 2.Syntax, their natural readings, natural examples

Semantics: A model $\mathrm{M}$ is a set $M$, together with interpretation functions

$$
\begin{aligned}
& {[[]]: P \rightarrow \mathrm{P}(M)} \\
& {[[]]: R \rightarrow \mathrm{P}(M \times M)}
\end{aligned}
$$

For each unary atom $p \in P,[[p]] \subseteq M$, and for each binary atom $r,[[r]] \subseteq M \times M_{R}$. We interpret set terms by subsets of $M$ in the following way:

$$
\begin{aligned}
& {[[\forall(r, q)]]=\{x \in M: \text { for all } v \in[[q]],(x, v) \in[[r]]\}} \\
& {[[\exists(r, q)]]=\{x \in M: \text { some } v \in[[q]],(x, v) \in[[r]]\}}
\end{aligned}
$$

Here is how set terms are read:

$$
\begin{aligned}
& \forall(r, b y): \text { those who } \mathrm{r} \text { all by } \\
& \forall(r, y): \text { those who } \mathrm{r} \text { all } \mathrm{y} \\
& \exists(r, b y): \text { those who } \mathrm{r} \text { some by }
\end{aligned}
$$


$\exists(r, y)$ : those who r some $\mathrm{y}$

Finally, we have the definition of truth in a model:

$$
\begin{aligned}
& M_{R} \mid=\forall(p, r(\forall, y)) \text { iff }[[p]] \subseteq[[r(\forall, y)]] \\
& M_{R} \mid=\forall(p, r(\exists, y)) \text { iff }[[p]] \subseteq[[r(\exists, y)]] \\
& M_{R} \mid=\exists(p, r(\exists, y) \text { iff }[[p]] \cap[[r(\exists, y)]] \neq \varnothing \\
& M_{R} \mid=\exists(p, r(\forall, y)) \text { iff }[[p]] \cap[[r(\forall, y)]] \neq \varnothing
\end{aligned}
$$

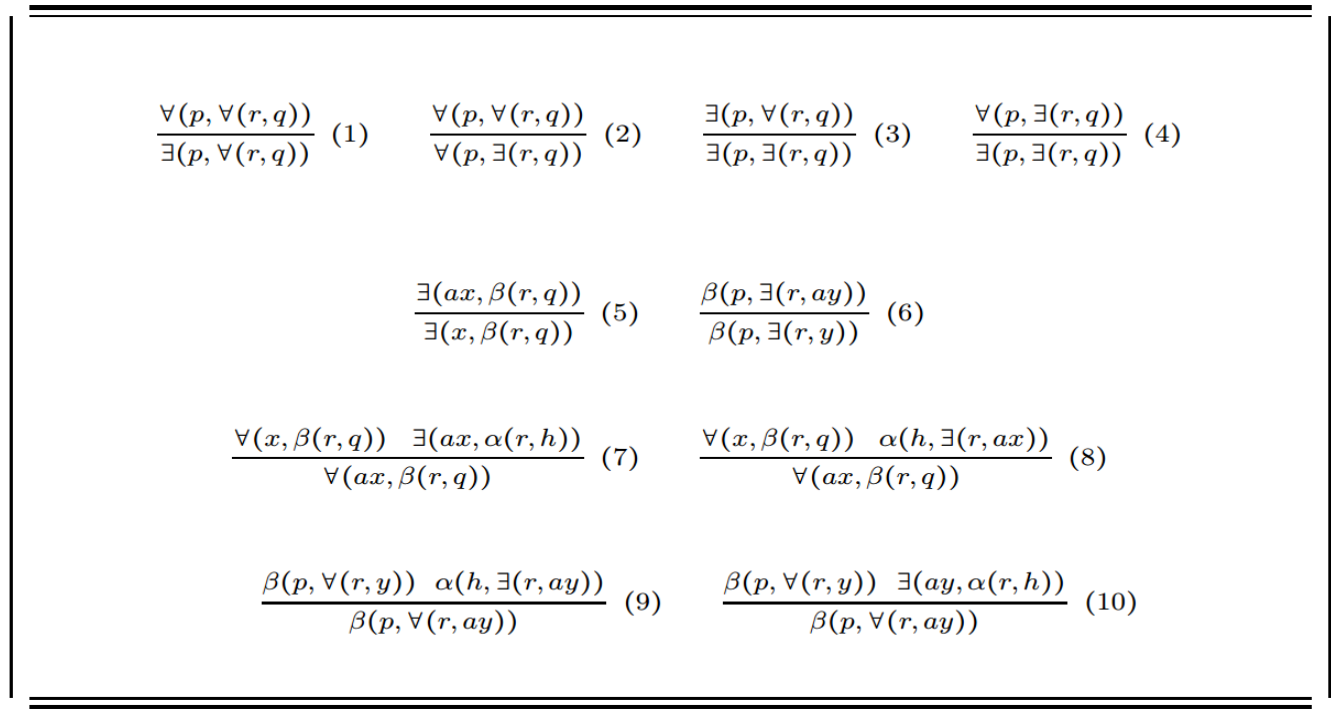

Fig. 2.Rules for $\mathrm{R}(\forall, \exists, \mathrm{IA}): p, h, q$ nouns, $x, y, z$ basic nouns, $\beta, \alpha \in\{\forall, \exists\}$

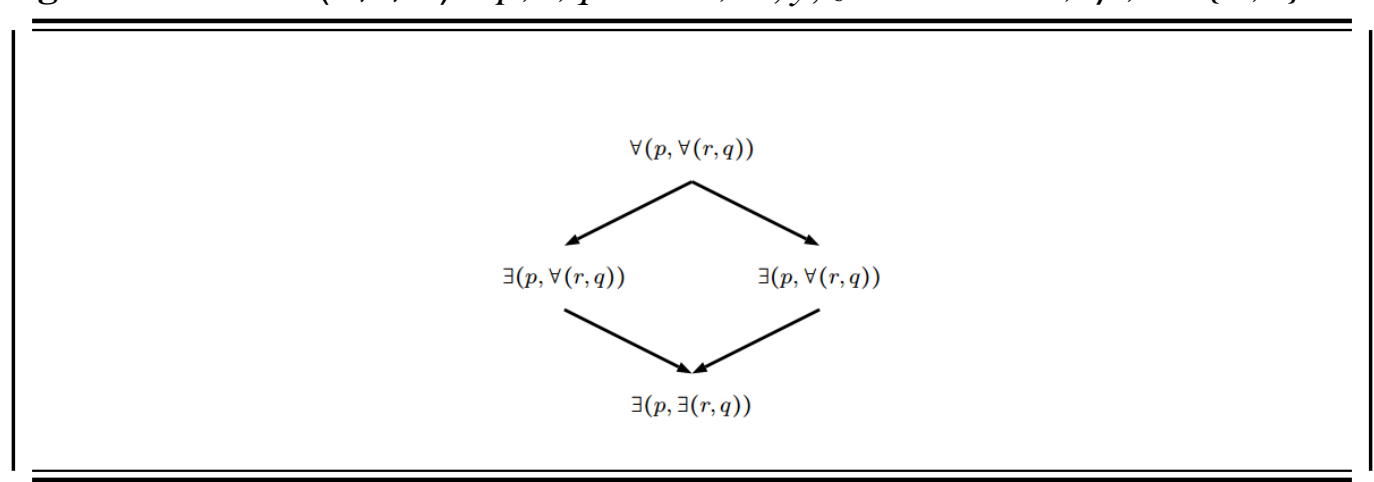

Fig. 3.Derivation diagram for rules (1), (2), (3), (4), (5). $p$ and $q$ are basic nouns or complex nouns.

Figure 2 indicates rule set of the logic. The rules (6) - (11) are abbreviated form of too many rules. For example, $\frac{\exists(a x, \forall(r, q))}{\exists(x, \forall(r, q))}$ and $\frac{\exists(a x, \exists(r, q))}{\exists(x, \exists(r, q))}$ are full form of $\frac{\exists(a x, \beta(r, q))}{\exists(x, \beta(r, q))}$. Figure 3 and Figure 4 shows that derivations of sentences from a sentence or sentences in the language of the logic. "If $\Gamma \mid-\varphi$, then $\Gamma \mid-\psi$ " is indicated by the arrows. The arrows do not work reverse direction. 


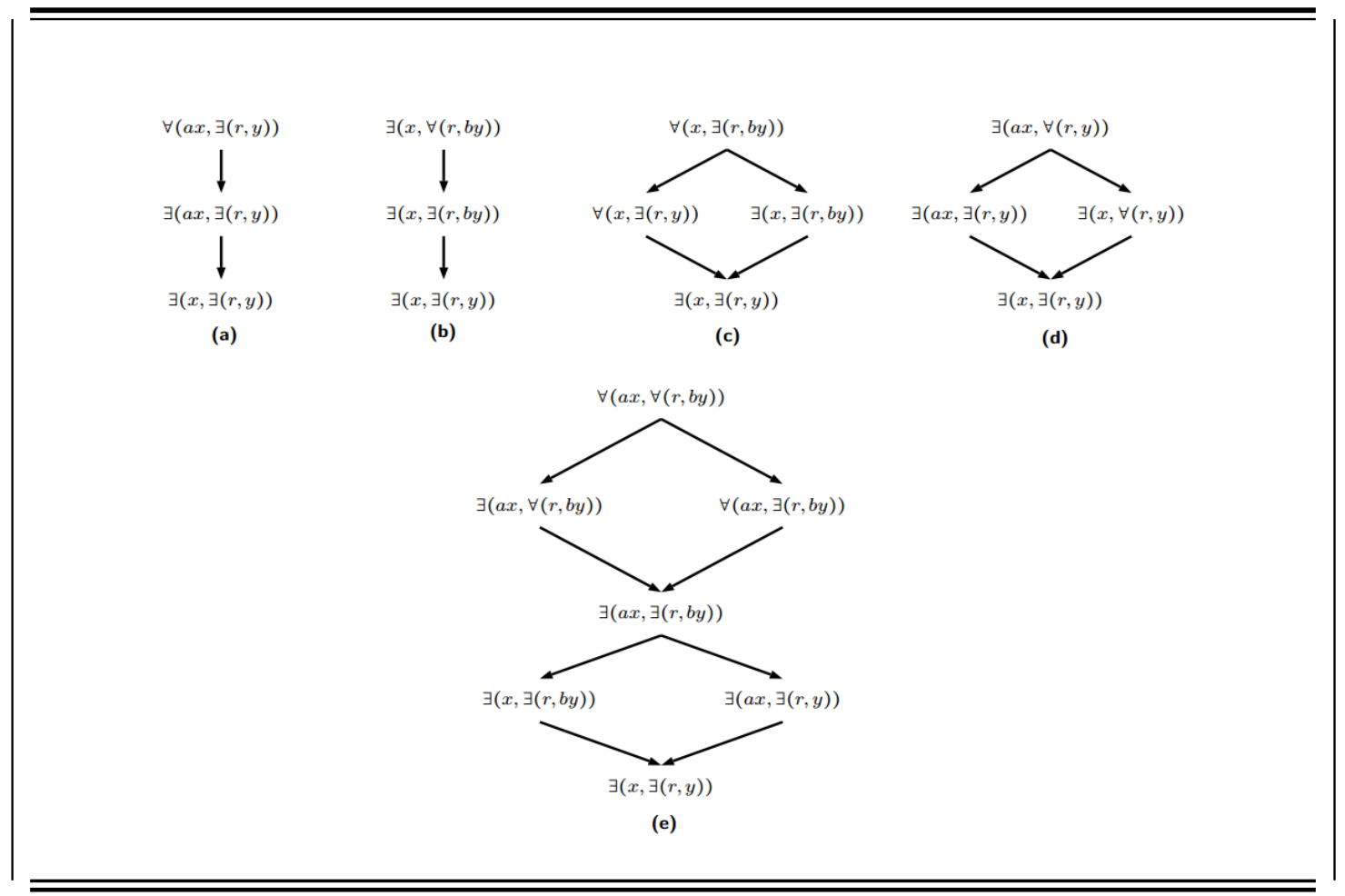

Fig. 4.Derivation diagrams for rules (6), (7), (8), (9), (10).

One of the main problems in logic is an algorithm to tell if $\Gamma \mid-\varphi$ or not. When one wants to check whether $\Gamma \mid-\exists(x, \exists(r, y))$ or not. All the arrows in Figure 3 and Figure 4 may be checked in the worst-case scenario for derivations in the logic. A model construction which tests being an element of a set and being a subset of a set is desired to not check the derivations in the scenario.

\subsection{Model Construction}

Definition 3.1. $P$ is a set of noun variables (complex or basic nouns), $\forall$ and $\exists$ arequantifiers in language of the logic. A set $\vec{P}$ consists of elements which accepted quantifiers in the language as subscript of nouns.

Example 3.2. If $P=\{x, y, a x\}$, then $\stackrel{P}{P}=\left\{x_{\forall}, y_{\forall}, a x_{\forall}, x_{\exists}, y_{\exists}, a x_{\exists}\right\}$

Definition 3.3. For a set of sentences $\Gamma, P_{\Gamma}$ is the set of nouns occurring in $\Gamma, R_{\Gamma}$ is the set of binary terms in $\Gamma$, and $\vec{P}_{\Gamma}$ is the set of elements of $P_{\Gamma}$ with their quantifiers.

Example 3.4. $\Gamma=\left\{\forall\left(x, \exists\left(r_{0}, y\right)\right), \exists\left(a x, \exists\left(r_{1}, b y\right)\right), \forall\left(c z, \forall\left(r_{1}, z\right)\right)\right\}$.

$$
R_{\Gamma}=\left\{r_{0}, r_{1}\right\}, P_{\Gamma}=\{a x, x, c z, b y, z\}, \vec{P}_{\Gamma}=\left\{x_{\forall}, c z_{\forall}, z_{\forall}, a x_{\exists}, y_{\exists}\right\}
$$

Definition 3.5. We define an translation from $\vec{P}$ to $\mathrm{P}(\vec{P})$ as the following:

$$
[]: \vec{P} \mapsto \mathrm{P}(\vec{P})
$$




$$
\begin{aligned}
& x_{\forall} \mapsto\left\{x_{\forall}, x_{\exists}\right\} \\
& x_{\exists} \mapsto\left\{x_{\exists}\right\} \\
& a x_{\forall} \mapsto\left\{a x_{\forall}, a x_{\exists}, x_{\exists}\right\} \\
& a x_{\exists} \mapsto\left\{a x_{\exists}, x_{\exists}\right\}
\end{aligned}
$$

Definition 3.6. We define two sets $\left[\vec{P}_{\Gamma}\right]=\left\{[i]\right.$ :for $\mathrm{i}$ in $\left.\vec{P}_{\Gamma}\right\}$ and $M^{+} \subseteq \vec{P} \times \vec{P} \times R$

Definition 3.7. Let $\Gamma$ be a set of sentences and $\Gamma_{V e c} \subseteq\left[\vec{P}_{\Gamma}\right] \times\left[\vec{P}_{\Gamma}\right] \times R_{\Gamma}$. We define a translation from $\Gamma$ to $\Gamma_{V e c}$.

$$
\begin{aligned}
& \Upsilon_{V}: \Gamma \mapsto \Gamma_{V e c} \\
& \alpha(p, \beta(r, q)) \mapsto\left(\left[p_{\alpha}\right],\left[q_{\beta}\right], r\right)
\end{aligned}
$$

Please notice that the translation is an one to one correspondence.

Remark 3.8. Notice that $\Gamma_{V e c} \subseteq M^{+}$.

Definition 3.9. Two elements $\left(\left[k_{\alpha}\right],\left[l_{\beta}\right], r_{0}\right)$ and $\left(\left[p_{\gamma}\right],\left[q_{\theta}\right], r_{1}\right)$ of $\Gamma_{V e c}$ are in the same equivalence class, if $k=a x$ or $k=x$ and $p=a x$ or $p=x$ and $l=b y$ or $l=y$ and $q=c z$ or $q=z$ and $r_{0}=r_{1}$ where $x, y, z$ are basic nouns and $a, b, c$ are intersective adjectives.

Remark 3.10. Two elements in $M^{+}$are in the same equivalence class, if the two elements that first two elements are represented by the same letters and last ones are the same. For instance, $\left(\left[p_{\alpha}\right],\left[q_{\beta}\right], r\right)$ and $\left(\left[p_{\gamma}\right],\left[q_{\theta}\right], r\right)$ are in the same equivalence class because first objects of two elements are denoted by $p$, second ones are $q$ and last ones are $r$.

Definition 3.11. A down-set of element $\left(\left[k_{\alpha}\right],\left[l_{\beta}\right], r_{0}\right)$ of $M^{+}$is a set $d_{\Downarrow}^{R}\left[\left(\left[k_{\alpha}\right],\left[l_{\beta}\right], r_{0}\right)\right]=\left\{\left(\left[p_{\alpha}\right],\left[m_{\beta}\right], r_{1}\right):\left[p_{\alpha}\right] \subseteq\left[k_{\alpha}\right]\right.$ and $\left[m_{\beta}\right] \subseteq\left[l_{\beta}\right]$ and $\left.r_{0}=r_{1}\right\} \quad$ and also we define $d_{\Downarrow}^{R}\left[M^{+}\right]=\left\{d_{\Downarrow}^{R}[i]: i \in M^{+}\right\}$.

\subsection{Constructing steps of $\stackrel{\triangleright}{M_{\Gamma}^{+}}$from $M_{\Gamma}^{+}$}

The following steps will be applied for every element of $M_{\Gamma}^{+}$. Note that we have first set $M_{\Gamma}^{+}=\Gamma_{V e c} \cap M^{+}$before applying the following steps.

1. If $\left(\beta,\left\{x_{\exists}, x_{\forall}\right\}, r\right) \in M_{\Gamma}^{+}$and $a x \in P_{\Gamma}$, then add $\left(\beta,\left\{a x_{\exists}, a x_{\forall}\right\}, r\right)$ to $M_{\Gamma}^{+}$.

2. If $\left(\left\{x_{\exists}, x_{\forall}\right\}, \gamma, r\right) \in M_{\Gamma}^{+}$and $a x \in P_{\Gamma}$, then add $\left(\left\{a x_{\exists}, a x_{\forall}\right\}, \gamma, r\right)$ to $M_{\Gamma}^{+}$.

3. If $\left(\left\{a x_{\exists}\right\}, \beta, r\right) \in M_{\Gamma}^{+}$or $\left(\left\{a x_{\exists}, a x_{\forall}\right\}, \beta, r\right) \in M_{\Gamma}^{+}$, then add $\left(\left\{x_{\exists}\right\}, \beta, r\right)$ to $M_{\Gamma}^{+}$.

4. If $\left(\beta,\left\{a x_{\exists}\right\}, r\right) \in M_{\Gamma}^{+}$or $\left(\beta,\left\{a x_{\exists}, a x_{\forall}\right\}, r\right) \in M_{\Gamma}^{+}$, then add $\left(\beta,\left\{x_{\exists}\right\}, r\right)$ to $M_{\Gamma}^{+}$.

5. If $\left(\left\{x_{\exists}, x_{\forall}\right\}, \beta, r\right) \in M_{\Gamma}^{+}$and $a x \in P_{\Gamma}$, then add $\left(\left\{a x_{\exists}, a x_{\forall}\right\}, \beta, r\right)$ to $M_{\Gamma}^{+}$.

6. Finally, the last step is to take $d_{\Downarrow}^{R}\left[M_{\Gamma}^{+}\right]$as $\stackrel{\triangleright}{\Gamma}_{\Gamma}^{+}$. 
Example 3.12. For a given $\Gamma=\left\{\forall\left(x, \exists\left(r_{0}, c y\right)\right), \exists\left(a x, \forall\left(r_{0}, d y\right)\right), \forall\left(k, \exists\left(r_{1}, c l\right)\right), \exists\left(e x, \exists\left(r_{2}, b k\right)\right)\right\}$,

$$
\begin{aligned}
& P_{\Gamma}=\{x, a x, c y, d y, k, c l, e x\}, \\
& \Gamma_{V e c}=\left\{\left(\left\{x_{\forall}, x_{\exists}\right\},\left\{c y_{\exists}, y_{\exists}\right\}, r_{0}\right),\left(\left\{a x_{\exists}, x_{\exists}\right\},\left\{d y_{\forall}, d y_{\exists}, y_{\exists}\right\}, r_{0}\right),\left(\left\{k_{\forall}, k_{\exists}\right\},\left\{c l_{\exists}, l_{\exists}\right\}, r_{1}\right),\left(\left\{e x_{\exists}, e_{\exists}\right\},\left\{b k_{\exists}, k_{\exists}\right\}, r_{2}\right)\right\}
\end{aligned}
$$

$M_{\Gamma}^{+}$is composed of all elements in Table 3 and Table 4 . The sign $\Downarrow$ indicates the sentences that can be derived from the sentence next to in the figures.

\begin{tabular}{|l|c|}
\hline \hline$\left(\left\{x_{\forall}, x_{\exists}\right\},\left\{c y_{\exists}, y_{\exists}\right\}, r_{0}\right) \Downarrow$ & $\left(\left\{a x_{\exists}, x_{\exists}\right\},\left\{d y_{\forall}, d y_{\exists}, y_{\exists}\right\}, r_{0}\right) \Downarrow$ \\
\hline$\left(\left\{x_{\exists}\right\},\left\{c y_{\exists}, y_{\exists}\right\}, r_{0}\right)$ & $\left(\left\{a x_{\exists}, x_{\exists}\right\},\left\{d y_{\exists}, y_{\exists}\right\}, r_{0}\right)$ \\
$\left(\left\{x_{\forall}, x_{\exists}\right\},\left\{y_{\exists}\right\}, r_{0}\right)$ & $\left(\left\{x_{\exists}\right\},\left\{d y_{\forall}, d y_{\exists}, y_{\exists}\right\}, r_{0}\right)$ \\
$\left(\left\{a x_{\forall}, a x_{\exists}\right\},\left\{y_{\exists}\right\}, r_{0}\right)$ since $a x \in P_{\Gamma}$ & $\left(\left\{x_{\exists}\right\},\left\{d y_{\exists}, y_{\exists}\right\}, r_{0}\right)$ \\
$\left(\left\{a x_{\exists}\right\},\left\{y_{\exists}\right\}, r_{0}\right)$ & $\left(\left\{a x_{\exists}, x_{\exists}\right\},\left\{y_{\exists}\right\}, r_{0}\right)$ \\
$\left(\left\{a x_{\exists}\right\},\left\{c y_{\exists}, y_{\exists}\right\}, r_{0}\right)$ & $\left(\left\{x_{\exists}\right\},\left\{y_{\exists}\right\}, r_{0}\right)$ \\
$\left(\left\{x_{\forall}, x_{\exists}\right\},\left\{y_{\exists}\right\}, r_{0}\right)$ & \\
$\left(\left\{x_{\exists}\right\},\left\{c y_{\exists}, y_{\exists}\right\}, r_{0}\right)$ & \\
$\left(\left\{x_{\exists}\right\},\left\{y_{\exists}\right\}, r_{0}\right)$ & \\
$\left(\left\{e x_{\forall}, e x_{\exists}\right\},\left\{y_{\exists}\right\}, r_{0}\right) \downarrow$ due to ex $x \in P_{\Gamma}$ & \\
\hline \hline
\end{tabular}

Table 3.Applying the constructing steps to $\left(\left\{x_{\forall}, x_{\exists}\right\},\left\{c y_{\exists}, y_{\exists}\right\}, r_{0}\right)$ and $\left(\left\{a x_{\exists}, x_{\exists}\right\},\left\{d y_{\forall}, d y_{\exists}, y_{\exists}\right\}, r_{0}\right)$

\begin{tabular}{|l|c|}
\hline$\overline{\left(\left\{k_{\forall}, k_{\exists}\right\},\left\{c l_{\exists}, l_{\exists}\right\}, r_{1}\right) \Downarrow}$ & $\left(\left\{e x_{\exists}, x_{\exists}\right\},\left\{b k_{\exists}, k_{\exists}\right\}, r_{2}\right) \Downarrow$ \\
\hline$\left(\left\{k_{\exists}\right\},\left\{c l_{\exists}, l_{\exists}\right\}, r_{1}\right)$ & $\left(\left\{x_{\exists}\right\},\left\{b k_{\exists}, k_{\exists}\right\}, r_{2}\right)$ \\
$\left(\left\{k_{\forall}, k_{\exists}\right\},\left\{l_{\exists}\right\}, r_{1}\right)$ & $\left(\left\{e x_{\exists}, x_{\exists}\right\},\left\{k_{\exists}\right\}, r_{2}\right)$ \\
$\left(\left\{k_{\exists}\right\},\left\{l_{\exists}\right\}, r_{1}\right)$ & \\
\hline \hline
\end{tabular}

Table 4.Applying the constructing steps to $\left(\left\{k_{\forall}, k_{\exists}\right\},\left\{c l_{\exists}, l_{\exists}\right\}, r_{1}\right)$ and $\left(\left\{e x_{\exists}, x_{\exists}\right\},\left\{b k_{\exists}, k_{\exists}\right\}, r_{2}\right)$

Definition 3.13. $\left[p_{\alpha}\right] \subseteq\left[k_{\alpha}\right]$ and $\left[m_{\beta}\right] \subseteq\left[l_{\beta}\right]$ and $r_{0}=r_{1}$ iff $\left(\left[p_{\alpha}\right],\left[m_{\beta}\right], r_{1}\right) \subseteq\left(\left[k_{\alpha}\right],\left[l_{\beta}\right], r_{0}\right)$

Theorem 3.14. $\Gamma \mid-\alpha(p, \beta(r, q))$ iff $\left(\left[p_{\alpha}\right],\left[q_{\beta}\right], r\right) \in M_{\Gamma}^{+}$, in other words, $\mathrm{M}_{R}=\left(M_{R},[[]]\right): \Leftrightarrow \mathrm{M}_{R}=\left(\stackrel{\triangleright}{M_{\Gamma}^{+}}, \in\right)$.

Proof 3.14. We will prove the theorem on noun complexity. Proofs for sentences having universal quantifiers with only basic nounswere already given in $R(\forall, \exists)$. Also, derivations of those sentences from a set of sentences are independent on existence of any other forms of sentences with or without adjectives. On the other hand, $M_{\Gamma}^{+}$in $\mathrm{R}(\forall, \exists, \mathrm{I} A)$ is a super set of $\stackrel{\triangleright}{\Gamma}_{\Gamma}^{+}$in $\mathrm{R}(\forall, \exists)$. We will prove the theorem considering those situations.

$(\Rightarrow)$ Supposing $\Gamma \mid-\alpha(p, \beta(r, q))$, we will show that $\left(\left[p_{\alpha}\right],\left[q_{\beta}\right], r\right) \in \stackrel{\triangleright}{M_{\Gamma}^{+}}$.

Case 1: $\Gamma \mid-\forall(a x, \forall(r, b y))$.If $\forall(a x, \forall(r, b y)) \in \Gamma$, then $\left(\left\{a x_{\forall}, a x_{\exists}, x_{\exists}\right\},\left\{b y_{\forall}, b y_{\exists}, y_{\exists}\right\}, r\right) \in M_{\Gamma}^{+}$， therefore, $\left(\left\{a x_{\forall}, a x_{\exists}, x_{\exists}\right\},\left\{b y_{\forall}, b y_{\exists}, y_{\exists}\right\}, r\right) \in{\stackrel{\triangleright}{M_{\Gamma}^{+}}}^{+}$. Suppose $\forall(a x, \forall(r, b y)) \notin \Gamma$ and $\Gamma \mid-\forall(x, \forall(r, y))$ 
and $a x \in P_{\Gamma}$ and $b y \in P_{\Gamma}$. We know that "if $\Gamma \mid-\forall(x, \forall(r, y))$, then $\left(\left\{x_{\forall}, x_{\exists}\right\},\left\{y_{\forall}, y_{\exists}\right\}, r\right) \in M_{\Gamma}^{+}$" by Proof 2.15. ( $\left.\left\{a x_{\forall}, a x_{\exists}, x_{\exists}\right\},\left\{b y_{\forall}, b y_{\exists}, y_{\exists}\right\}, r\right)$ is added to $M_{\Gamma}^{+}$from the construction (1) and (2). Finally, $\left(\left\{a x_{\forall}, a x_{\exists}, x_{\exists}\right\},\left\{b y_{\forall}, b y_{\exists}, y_{\exists}\right\}, r\right) \in M_{\Gamma}^{+}$.

Please note that it is hold for all derivable sentences from $\forall(a x, \forall(r, b y))$ since all down sets of $\left(\left\{a x_{\forall}, a x_{\exists}, x_{\exists}\right\},\left\{b y_{\forall}, b y_{\exists}, y_{\exists}\right\}, r\right)$ are contained by the construction.

Case 2: $\Gamma \mid-\exists(a x, \forall(r, b y))$. If $\exists(a x, \forall(r, b y)) \in \Gamma$, it is clear. Otherwise, there is a proof tree whose root is $\exists(a x, \forall(r, b y))$. There are some cases for this derivation as the follows:

(a) If $\Gamma \mid-\forall(a x, \forall(r, b y))$, we proved and mentioned it in Case 1.

(b) If $\Gamma \mid-\forall(x, \forall(r, b y))$ and $a x \in P_{\Gamma}$, then $\Gamma \mid-\forall(a x, \forall(r, b y))$ again.

(c) If $\Gamma \mid-\forall(a x, \forall(r, y))$ and $b y \in P_{\Gamma}$, then $\Gamma \mid-\forall(a x, \forall(r, b y))$ again (by Case 1$)$.

(d) If $\Gamma \mid-\forall(x, \forall(r, y))$ and $a x, b y \in P_{\Gamma}$, then $\Gamma \mid-\forall(a x, \forall(r, b y))$ again (by Case 1$)$.

Case 3: $\Gamma \mid-\forall(a x, \exists(r, b y))$ is routine.

Case 4: $\Gamma \mid-\exists(a x, \exists(r, b y))$ is routine.

Case 5: $\Gamma \mid-\exists(x, \exists(r, y))$. If $\exists(x, \exists(r, y)) \notin \Gamma$, there are possibly an awful lot of proof trees whose roots are $\exists(x, \exists(r, y))$ as can be seen in Figure 4. Starting the proofs as we mentioned, for all sentences which derive $\exists(x, \exists(r, y))$ are hold. If no sentences of $\Gamma$ derives $\exists(x, \exists(r, y))$ except itself, it contradicts our $\exists(x, \exists(r, y)) \notin \Gamma$. Hence, if there exists at least one sentence which derives $\exists(x, \exists(r, y))$, then $\left(\left[x_{\exists}\right],\left[y_{\exists}\right], r\right)$ must be in ${\stackrel{\triangleright}{M^{+}}}^{+}$.

Other proofs are routine.

$(\Leftarrow)$ We will show that $\Gamma \mid-\alpha(p, \beta(r, q))$ supposing $\left(\left[p_{\alpha}\right],\left[q_{\beta}\right], r\right) \in M_{\Gamma}^{+}$.

If any $\left(\left[p_{\alpha}\right],\left[q_{\beta}\right], r\right)$ in $\Gamma_{V e c}$, the proof is easy. Otherwise, we will use the down-set definition and property of one to one correspondence of $\Gamma_{V e c}$.

Let be $\left(\left[a x_{\forall}\right],\left[b y_{\forall}\right], r\right)$ in $M_{\Gamma}^{+}$. Suppose that $\left(\left[a x_{\forall}\right],\left[b y_{\forall}\right], r\right) \notin \Gamma_{V e c} \quad$ (otherwise, $\forall(a x, \forall(r, b y)) \in \Gamma$, therefore, $\Gamma \mid-\forall(a x, \forall(r, b y)))$. Then there is a $\left(\left[p_{\alpha}\right],\left[q_{\beta}, r\right]\right)$ where $\left(\left[a x_{\forall}\right],\left[b y_{\forall}\right], r\right)$ is a an element of $d_{\Downarrow}^{R}\left[\left(\left[p_{\alpha}\right],\left[q_{\beta}, r\right]\right)\right]$. So, $\left(\left[p_{\alpha}\right],\left[q_{\beta}\right], r\right)$ must be in $\Gamma_{V e c}$ since $\Upsilon_{V}$ is an one to one correspondence. Hence, $\alpha(p, \beta(r, q)) \in \Gamma$. Finally, $\alpha(p, \beta(r, q))$ follows $\alpha(p, \beta(r, q))$. Other proofs are routine.

Theorem 3.15. $\Gamma \mid-\varphi$ iff there exists at least one $\psi$ such that $[\varphi] \subseteq[\psi]$ in $M_{\Gamma}^{+}$.

Proof 3.15. We saw that there is at least one upper set of $\varphi$ to derive it from $\Gamma$ or a sentence $\psi$ due to the definitions $\Upsilon_{V}$ and down-sets in the sufficient condition of Theorem 3.15.

Corollary 3.16. Let $\Gamma$ be set of sentences in $\mathrm{R}(\forall, \exists) .\left(M_{\Gamma},[[]]\right),\left(\stackrel{\triangleright}{M_{\Gamma}^{+}}, \in\right)$ and $\left(\stackrel{\triangleright}{M_{\Gamma}^{+}}, \subseteq\right)$ are equivalent models.

Corollary 3.17. Let $\Gamma$ be set of sentences in $\mathrm{R}(\forall, \exists) .\left(M_{\Gamma},[[]]\right),\left(M_{\Gamma}^{+}, \in\right)$ and $\left(M_{\Gamma}^{+}, \subseteq\right)$ are equivalent models. 


\section{Conclusion}

This paper has presented two logical systems and their set-theoretic semantics. The smaller system consists of transitive verbs and quantifiers. The bigger system is an extension of the small one which is restricted to intersective adjectives. The logical systems have three equivalent set-theoretic models. $\left(\stackrel{\triangleright}{M_{\Gamma}^{+}}, \in\right)$ and $\left(\stackrel{\triangleright}{M_{\Gamma}^{+}}, \subseteq\right)$ provide simplicity for checking derivability and non-derivability of a sentence from a set of sentences and also truth and falsity of a sentence in models of the logics because the models are built on the idea of equivalence class, being elements of a set and also testing whether a subset or not.

We hope that logico-linguists, applied and theoretical computer scientists, and pure and applied logicians might be interested in results in this paper.

\section{Acknowledgments}

The authors would like to thank the anonymous reviewers for their helpful comments. They would also like to thank the editors for their open-hearted comments and support during the review process.

\section{References}

1. Benthem van, J. Questions about quantifiers, Journal of Symbolic Logic, 49, 2, 1984, pp. 443-466.

2. Black, M. A New Method of Presentation of the Theory of the Syllogism, The Journal of Philosophy, 42, 17, 1945, pp. 449-455.

3. Bocharov, V. A. Boolean algebra and syllogism, Synthese, 66, 1, 1986, pp. 35-54.

4. Corcoran J., Completeness of an ancient logic, Journal of Symbolic Logic, 37, 1972, pp. 696-702.

5. D'Alfonso, D. The square of opposition and generalized quantifiers In J. Y. Béziau \& D. Jacquette (eds.), Around and Beyond the Square of Opposition. Studies in Universal Logic, Basel:Springer, 2012, pp. 219-227.

6. De Morgan, A. Formal logic: or, the calculus of inference, necessary and probable, Taylor and Walton, 1847.

7. Eijck van, J. Generalized quantifiers and traditional logic, In J. van Benthem \& A. T. Meulen (eds.), Generalized quantifiers in natural language, vol. 4, Walter de Gruyter,1985, pp. 1-19.

8. Eijck van, J.Syllogistics = monotonicity + symmetry + existential import, May, 2005, preprint.

9. Eijck van, J. Natural Logic for Natural Language, In B. D. ten Cate \& H.W. Zeevat (eds.), Logic, Language, and Computation, TbiLLC 2005, Lecture Notes in Computer Science, vol. 4363, Berlin, Heidelberg:Springer, 2007.

10. Ivanov, N. \& D.Vakarelov. A system of relational syllogistic incorporating full Boolean reasoning, Journal of Logic, Language and Information, 21(4), 2012, pp. 433-459.

11. Lukasiewicz, J. Aristotle's syllogistic from the standpoint of modern formal logic, Oxford University Press, 1957.

12. Moss, L. S. Completeness theorems for syllogistic fragments, In F.Hamm \& S. Kepser (eds.), Logics for Linguistic Structures, 2008, pp. 143-173.

13. Moss L.S. Intersecting Adjectives in Syllogistic Logic, In C. Ebert, G. Jäger \& J. Michaelis (eds), The Mathematics of Language. Lecture Notes in Computer Science, vol. 6149, Berlin, Heidelberg: Springer, 2010, pp. 223-237.

14. Moss L. S. Syllogistic Logic with Complements, In J. van Benthem, A. Gupta \& E. Pacuit (eds), Games, Norms and Reasons. Synthese Library. Studies in Epistemology, Logic, Methodology, and Philosophy of Science, vol. 353, Dordrecht: Springer, Springer Netherlands, 2011, pp. 179-197.

15. Moss L. S. Syllogistic logics with verbs, Journal of Logic and Computation, 20 (4),2010, pp. 947-967. 
16. Peirce C. S. On the algebra of logic, American Journal of Mathematics, 3(1), 1880, pp. 15-57.

17. Pratt-Hartmann I. \& L. S.Moss. Logics for the relational syllogistic, The Review of Symbolic Logic, 2 (04), 2009, pp. 647-683.

18. Schumann, A. \& L. Akimova. Syllogistic system for the propagation of parasites, The case of Schistosomatidae (Trematoda: Digenea), Studies in Logic, Grammar and Rhetoric, 40 (53), 2015.

19. Schumann A. On Two Squares of Opposition: the Lesniewskis Style Formalization of Synthetic Propositions, Acta Analytica, 28, 1, 2013, pp. 71-93.

20. Sotirov V. Arithmetizations of syllogistic a la Leibniz, Journal of Applied Non-Classical Logics, 9 , 2-3, 1999, pp. 387-405.

21. Topal, S. Equivalential Structures for Binary and Ternary Syllogistics, Journal of Logic, Language and Information, 2017, https://doi.org/10.1007/s10849-017-9260-4

22. Westerståhl, D. On the Aristotelian square of opposition, Kapten Mnemos Kolumbarium, en festskrift med anledning av Helge Malmgrens 60-årsdag, 2005. 